\title{
Removal of the Basal Lamina in vivo Reveals Growth Cone-Basal Lamina Adhesive Interactions and Axonal Tension in Grasshopper Embryos
}

\author{
Maureen L. Condic ${ }^{1}$ and David Bentley² \\ 'Neurobiology Group and 2Department of Molecular and Cell Biology, University of California, Berkeley, California 94720
}

The Ti1 afferent neurons are the first cells to undergo axonogenesis in embryonic grasshopper limbs. The Ti1 growth cones migrate between the limb epithelium and its basal lamina. We have investigated the nature of growth conebasal lamina interactions in vivo by removing the basal lamina with mild enzymatic digestion. Treatment with elastase, ficin, or papain removes the basal lamina when viewed in scanning electron microscopy. Trypsin and chymotrypsin leave the basal lamina intact.

If the basal lamina is removed after the Ti1 growth cones have extended over intrasegmental epithelium but are not yet in contact with either differentiated segment boundaries or neurons, the growth cones retract to the cell somata. If the basal lamina is removed by elastase, and the $\mathrm{Ti} 1$ neurons are allowed to extend axons after treatment, a second elastase digestion does not cause the axons to retract. It is therefore unlikely that axon retraction is due to general proteolysis. These results suggest that if $\mathrm{Ti} 1$ growth cones have initially extended in the presence of an intact basal lamina, they are dependent on the lamina to remain extended over this region of the limb.

The retraction of the Ti1 axons after removal of the basal lamina is inhibited by cytochalasin $D$, suggesting that microfilament-based cytoskeletal components underlie this event. This result indicates that the axons are under tension in vivo. The ability of the Ti1 growth cones to resist axonal tension suggests that adhesive interactions between the growth cones and the basal lamina underlie normal axon outgrowth in vivo.

Growth cone adhesion to basal lamina and extracellular matrix proteins may constitute an important element of axon guidance during development (Hay, 1981; Jessell, 1988) and neuronal regeneration (Sanes and Chiu, 1983). It is well established that neuronal growth cones in vitro recognize and adhere to a number of extracellular matrix proteins. A variety of cellular receptors that are specific for extracellular matrix components have been

Received Sept. 30, 1988; revised Dec. 20, 1988; accepted Jan. 20, 1989.

We thank Dr. P. Letourneau for suggesting the cytochalasin experiment, A. Toroian-Raymond for suggesting the sequential elastase treatments and for assistance with SEM preparations, Dr. L. Fessler for providing a purified collagenase preparation, and Dr. D. Fristrom and Dr. H. J. Yost for their critical reading of the manuscript. This work was supported by an NSF predoctoral grant and an NIH predoctoral training grant (T32-GM07048-14) to M.L.C.; NIH Jacob Javits grant (NS09094-19) and March of Dimes Birth Defects Foundation grant (1-1089) to D.B.

Correspondence should be addressed to Maureen L. Condic at the above address. Copyright (C) 1989 Society for Neuroscience $0270-6474 / 89 / 082678-09 \$ 02.00 / 0$ described, many of which are believed to function as cell-substrate adhesion molecules (Obrink, 1986; Hynes, 1987; Jessell, 1988). Whether growth cones establish adhesive interactions with extracellular matrix components in vivo has not been determined. Embryonic grasshopper limbs provide a relatively simple system in which the interactions of pioneer growth cones and basal lamina can be investigated in vivo.

At the time of pioneer axonogenesis, the embryonic grasshopper limb bud consists of a simple epithelial cone surrounding a monolayer of undifferentiated mesodermal cells with a basal lamina separating the mesodermal and epithelial cell layers (Wigglesworth, 1953; Ashhurst, 1965, 1982). 'The basal lamina is thought to be deposited largely by epithelial cells, but mesodcrmal hemocytes also contribute to its composition (Ball et al., 1987; Mirre et al., 1988). Basal laminae of insects contain a number of molecular components common to vertebrate laminae, including collagen type IV (Blumberg et al., 1987), laminin (Fessler et al., 1987; Montell and Goodman, 1988), fibronectin (Grateios et al., 1988), and an entactin/nidogen-like compound (Blumberg et al., 1987). In addition, receptors homologous to the vertebrate integrin family have recently been identified in insects (Bogaert et al., 1987; Leptin et al., 1987; MacKrell et al., 1988).

During grasshopper embryonic development, the Til pioneer axons establish the first neuronal pathway observed in limb buds (Bate, 1976; Keshishian, 1980). At 30\% of development, the Til cells arise from an apical to basal division of an epithelial mother cell at the tip of the limb (Keshishian, 1980; Lefcort and Bentley, 1989). The neurons then emerge from the epithelium and extend axons between the basal lamina and the basal end feet of the limb epithelium (Bate, 1976; Keshishian and Bentley, 1983; Lefcort and Bentley, 1987). The growth cones extend numerous filopodia between the epithelial cells and through the basal lamina into the mesodermal cell layer (Caudy and Bentley, 1986a).

In the work reported here we have examined the Til neurons in vivo and determined the effects of removing the basal lamina when the Til growth cones were in contact with basal lamina and intersegmental epithelium.

\section{Materials and Methods}

Embryos of Schistocerca americana were obtained from a colony maintained at the University of California, Berkeley. They were staged by the percentage of total embryonic development completed (Bentley et al., 1979; Caudy and Bentley, 1986a). Limb axes, specific neurons, and segment boundaries are named as in Caudy and Bentley (1986a; for depiction of embryonic vs adult limb axes, see Ball and Goodman, 1985). 
Table 1. Enzyme effects on basal lamina and on neuronal disposition

\begin{tabular}{lllll} 
Enzyme & $\begin{array}{l}\text { Embryo } \\
\text { stage } \\
(\%)^{a}\end{array}$ & $\begin{array}{l}\text { Enzyme } \\
\text { conc. (\%) }\end{array}$ & $\begin{array}{l}\text { Disruption of } \\
\text { basal lamina } \\
(n)^{b}\end{array}$ & $\begin{array}{l}\text { Growth } \\
\text { cone } \\
\text { retraction }\end{array}$ \\
\hline Control & 32.5 & $-{ }^{d}$ & $0(13)$ & $0 / 31$ \\
& 34.5 & - & $0(4)$ & - \\
Chymotrypsin & 36.5 & - & $0(7)$ & - \\
Trypsin & 32.5 & $0.005-0.01$ & $0(8)$ & $0 / 13$ \\
& 32.5 & $0.01-0.02$ & $0 /+(8)$ & $0 / 12$ \\
Papain & 34.5 & $0.02-0.04$ & $0 /+(6)$ & - \\
Elastase & 32.5 & $0.025-0.05$ & $++(5)$ & $10 / 18$ \\
& 32.5 & $0.04-0.1$ & $+++(8)$ & $11 / 15$ \\
& 34.5 & $0.1-0.25$ & $+++(6)$ & - \\
Ficin & 36.5 & $0.1-0.25$ & $+++(10)$ & - \\
& 32.5 & $0.01-0.02$ & $+++(5)$ & $9 / 20$ \\
& 36.5 & $0.02-0.04$ & $+++(8)$ & - \\
\hline
\end{tabular}

${ }^{a}$ Percentage of complete development $( \pm 0.5)$.

${ }^{h}$ Evaluated in SEM: 0, no effect; + , slight removal; ++ , less than complete removal; +++ , complete removal.

' Number of embryos with $\geq 50 \%$ axon pairs affected per total number of embryos examined.

${ }^{4}$ Not applicable.

Enzyme treatments. Trypsin (T-8253), elastase (E-0258), chymotrypsin (C-4129), ficin (F-6008), and papain (P-4762) were obtained from Sigma. Collagenase (Sigma, C-0773) did not give consistent results on grasshopper basal lamina. The enzymes were stored as $0.5 \%$ solutions in saline $\left(\mathrm{NaCl}, 150 \mathrm{~mm} ; \mathrm{CaCl}_{2}, 4 \mathrm{~mm} ; \mathrm{KCl}, 10 \mathrm{~mm}, \mathrm{MgCl}_{2}, 2 \mathrm{~mm}\right.$; TES, $5 \mathrm{~mm}$; sucrose, $140 \mathrm{~mm} ; 0.1 \% \mathrm{BSA}, \mathrm{pH} 7.2$ ) at $-20^{\circ} \mathrm{C}$. For each enzyme considered, comparable effective enzyme concentrations were determined as follows: embryos were dissected in saline, opened dorsally, and exposed to a range of enzyme concentrations for $2.5-3 \mathrm{hr}$ at $30^{\circ} \mathrm{C}$. The highest concentration in which the limb epithelium remained intact over this period was determincd for each lot of enzyme purchased. All subsequent experiments were performed at one-fifth the maximum concentration determined for that lot. This value varied by approximately a factor of 2 between enzyme lots. The range of concentrations used for each enzyme is reported in Table 1. Note: When BSA was excluded from the media the enzyme concentrations required to elicit an effect on the tissue were consistently lower, but the relative effects of the different enzymes were not altered.

Scanning electron microscopy. To determine the effects of different enzymes on the basal lamina, the mesoderm was removed from the right metathoracic leg with a suction pipette (Lefcort and Bentley, 1987), and the embryo was allowed to recover for $1 \mathrm{hr}$ in saline at $30^{\circ} \mathrm{C}$. It was then exposed to either enzyme or saline solution for $1.5-2.5 \mathrm{hr}$, fixed immediately for $2 \mathrm{hr}$ in Karnovski's fixative ( $2 \%$ glutaraldehyde, $2.25 \%$ paraformaldehyde, in $0.1 \mathrm{M}$ sodium cacodylate buffer, $\mathrm{pH} 7.5$ ), postfixed for $2 \mathrm{hr}$ in $2 \% \mathrm{OsO}_{4}$ (in $0.1 \mathrm{~m}$ sodium cacodylate buffer, $\mathrm{pH}$ 7.5), dehydrated, critical point-dried, and mounted on a stub. Note: The shorter incubation period used for SEM preparations reflects the shorter amount of time required for the enzyme solutions to diffuse into limbs from which the mesoderm had been removed. The posterior face of the metathoracic leg was fractured off with a sharpened tungsten needle (Fig. $1 B$ ). The preparation was then coated in gold-palladium and viewed in a ISI-DS-130 SEM. Legs from 88 embryos were examined (Table 1).

Immunofluorescent labeling of neurons in whole-mounted embryos. The effects of enzyme incubation on the disposition of the neurons was determined in immunofluorescence-labeled embryos. Embryos were exposed to enzyme as described above (see Table 1). Immediately following incubation, the embryos were fixed overnight in $4 \%$ formaldehyde in saline (without sucrose). Neurons were then labeled with anti-HRP antibodies according to the protocol of Caudy and Bentley (1986a). These antibodies selectively label insect neurons (Jan and Jan, 1982; Snow et al., 1987). Embryos were viewed and photographed in wholemount with a Zeiss epifluorescence microscope. Over 300 embryos were examined, and data from 109 are presented in Table 1.

Quantification of growth cone retraction. To quantify Til growth cone retraction, embryos from a single clutch of eggs were staged to $32 \pm$
Table 2. Response of the Ti1 growth cones to serial elastase treatment

\begin{tabular}{lll} 
Condition & $\begin{array}{l}\text { Number of } \\
\text { limbs } \\
(n)\end{array}$ & $\begin{array}{l}\text { Limbs } \\
\text { without } \\
\text { axons }^{\prime \prime}(\%)\end{array}$ \\
\hline $\begin{array}{l}\text { Control } \\
\text { Elastase-treated }\end{array}$ & $\begin{array}{l}72(12) \\
54(9)\end{array}$ & $\begin{array}{l}17 \\
20\end{array}$ \\
$\begin{array}{l}\text { Control: post } \\
\text { elastase treatment } \\
\text { Elastase-treated: post } \\
\text { second elastase treatment }\end{array}$ & $84(14)$ & 45 \\
\hline
\end{tabular}

Embryos prior to Til axonogenesis were incubated with saline (control) or $0.04 \%$ elastase (elastase-treated) for $3 \mathrm{hr}$, then rinsed extensively and cultured for 16-17 $\mathrm{hr}$. Control and clastase-treated embryos were then incubated $3 \mathrm{hr}$ with 0.04 $0.1 \%$ elastase.

${ }^{a}$ Number of limbs examined in each condition; with number of embryos given in parentheses.

${ }^{\text {h }}$ Percentage of limbs with axons $\leq 1$ cell diameter or with clear evidence of growth cone condensation.

$0.5 \%$ of devclopment and incubated in clastase $(0.04 \%)$, trypsin $(0.01 \%)$, or saline as described above. Embryos were then fixed and labeled with anti-HRP antibodies. Measurements were made using an ocular micrometer. The length of the axon was determined by measuring from the midpoint of the Til cell body pair to the most proximal process of their growth cones that was not a filopodium. The distance between this point on the growth cones and the center of the Cxl cell body pair was also recorded. The distance between the Cx1 neurons and the Ti1 somata was measured as a straight line between the centers of the cell body pairs. Only legs in which both the Ti1 and Cxl cells were clearly visible with the anti-HRP antibody were measured (Fig. 4).

Embryo culture. Eggs were sterilized in $0.02 \%$ benzethonium chloride (in $70 \%$ ethanol) and dissected in saline under sterile conditions. Embryos were incubated for $3 \mathrm{hr}$ in either saline or $0.04 \%$ elastase as described above, washed cxtensively for $30 \mathrm{~min}$, then cultured at $30^{\circ} \mathrm{C}$ in a $\mathrm{CO}_{2}$ incubator in the following solution: RPMI 1640 (Gibco, N.Y.), $0.2 \%$ sodium bicarbonate, $1 \mu \mathrm{M}$ oxaloacetic acid, $0.45 \mathrm{~mm}$ sodium pyruvate, $2 \mathrm{~mm}$ L-glutamine, $0.45 \% \mathrm{D}(+)$ glucose, $0.02 \mathrm{IU} / \mathrm{ml}$ insulin, 3 $\mu \mathrm{M}$ b-ecdysterone (Sigma), $50 \mu \mathrm{g} / \mathrm{ml}$ gentamicin, $130 \mathrm{~mm}$ sucrose, 10 mM xylose, $0.1 \mu \mathrm{M}$ retinoic acid, $5 \mathrm{~mm}$ TES, and 0.1\% BSA, pH 7.0. Embryos were cultured for 16-17 hr, after which some animals from both groups were fixed to confirm that neuronal outgrowth had occurred. The remaining control and elastase-treated embryos were subjected to protease digestion for $3 \mathrm{hr}$ using $0.04-0.1 \%$ elastase, fixed, and labeled with anti-HRP antibodies. The number of limbs from pre- and posttreatment conditions with axons less than one cell diameter in length or with a condensed mass of cellular material at the proximal pole of the axons were recorded. The results of 4 similar experiments are reported in Table 2.

Table 3. Elastase-induced axon retraction in the presence of cytochalasin D

\begin{tabular}{llc} 
Condition & $\begin{array}{l}\text { Number } \\
\text { of limbs }\end{array}$ & $\begin{array}{l}\text { Limbs } \\
\text { without } \\
\text { axons }^{b}(\%)\end{array}$ \\
\hline Control & $30(5)$ & 3 \\
Cytochalasin D & $48(8)$ & 8 \\
Cytochalasin D and elastase & $30(5)$ & 20 \\
Elastase & $36(6)$ & 56
\end{tabular}

Embryos at the $32 \%$ stage were incubated with either saline, cytochalasin D at $0.1-0.5 \mu \mathrm{M} / \mathrm{ml}$, elastase at $0.04 \%$ with cytochalasin $\mathrm{D}$, or elastase alone for 2.5 hr.

${ }^{a}$ Number of limbs examined, with number of embryos given in parentheses.

${ }^{\text {h }}$ Limbs with axons $<1$ cell diameter in length or with clear evidence of growth cone condensation. 
Figure 1. Scanning electron micrographs of metathoracic limbs with and without mesoderm. Limbs at $32 \%$ of development were fractured longitudinally to remove the posterior face of the limb. $A$, Control limb with epithelium $(e)$ and intact mesodermal cells $(m)$ visible. A portion of the dense basal lamina apposed to the basal endfeet of the epithelium is visible at the arrowhead. $B$, Limb from which the mesoderm has been removed. Smooth basal lamina $(b l)$ entirely covers the lumenal surface of the epithelium. Profiles of the Til somata beneath the basal lamina are indicated by the arrow. The apical endfeet of epithelial cells can be seen at $a$. Dorsal is up; distal is to the right. Scale bar, $25 \mu \mathrm{m}$.
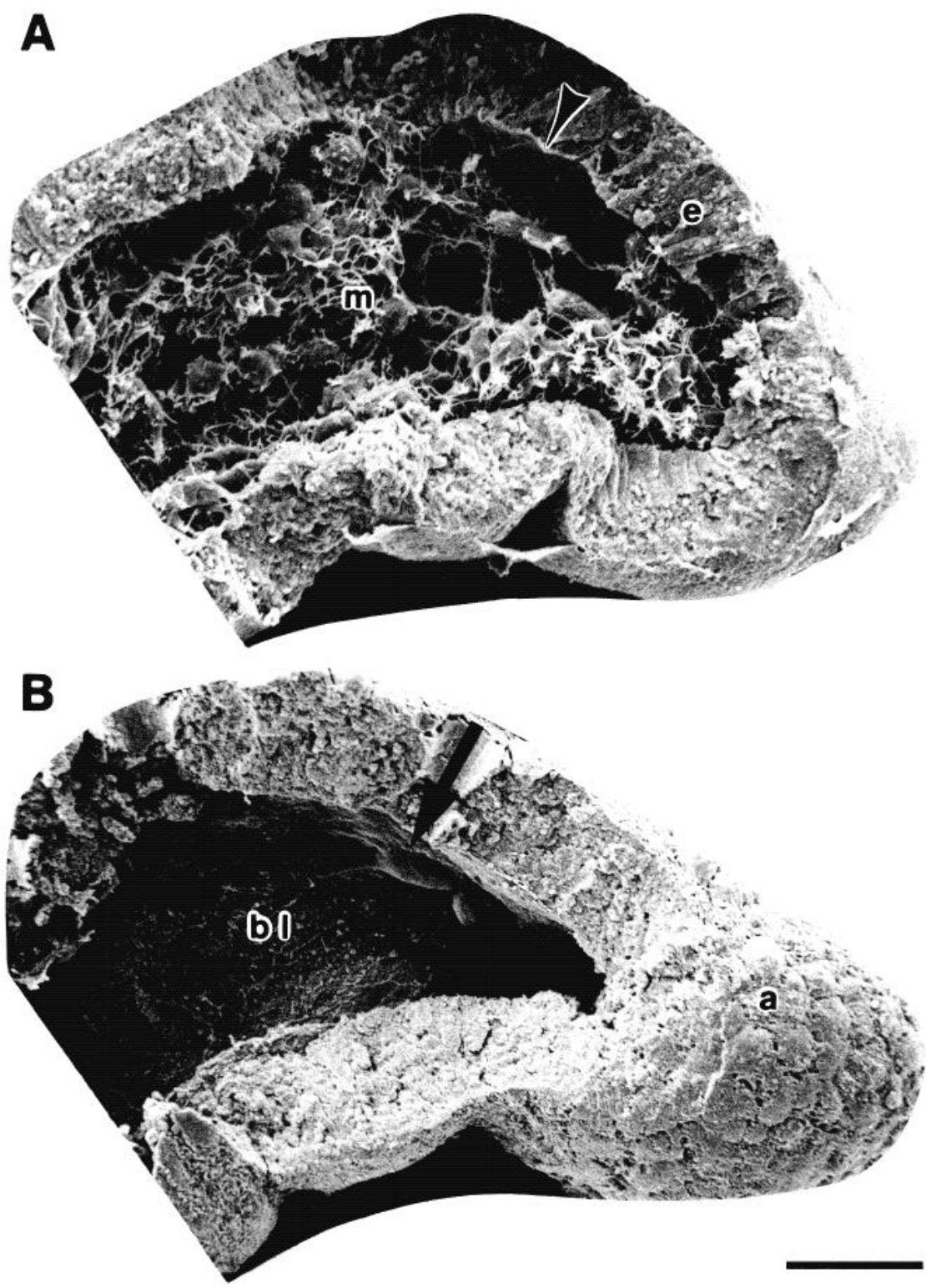

Cytochalasin treatment. Embryos at the $32 \%$ stage were incubated for $3 \mathrm{hr}$ in either saline or $0.04 \%$ elastase as described above. Cytochalasin D (Sigma C-8273) was stored as a $1 \mathrm{mg} / \mathrm{ml}$ stock solution in DMSO at $-20^{\circ} \mathrm{C}$. At the beginning of the incubation period, cytochalasin $\mathrm{D}$ was added to the enzyme or control solutions at a final concentration of $0.1 \mu \mathrm{g} / \mathrm{ml}$ or $0.5 \mu \mathrm{g} / \mathrm{ml}$. The embryos were fixed after $3 \mathrm{hr}$, stained with the anti-HRP antibody, and viewed in whole-mount. The number of limbs with axons less than one cell diameter in length or with evidence of condensed cellular material in the region of the growth cones were recorded. The results of 3 similar experiments are reported in Table 3.

\section{Results}

Embryonic grasshopper limb buds at the $32 \%$ stage consist of an epithelial monolayer (and associated basal lamina) surrounding a loose arrangement of mesenchymal cells (Fig. 1A). At this stage, the Til neurons have emerged from the epithelium and begun axonogenesis. The mesodermal cells can be removed from the limb bud by inserting a pipette through the dorsal closure into the lumen of the limb and applying mild suction (Lefcort and Bentley, 1987). This procedure affords a view of the basal surface of the epithelium (Fig. $1 B$ ). In normal embryos observed in SEM, the appearance of the basal lamina changed substantially over the range of developmental stages observed and varied considerably between individuals at the same stage of development. The basal lamina of the youngest embryos examined (the $32 \%$ stage) most frequently appeared matted and fibrous (Figs. $1 B, 2 A$ ). The epithelial endfeet and the Til somata and axons are completely obscured by the basal lamina at this age (Fig. 1B). At successively older stages, the lamina became increasingly smooth and condensed in appearance. Various proteolytic enzymes were tested for their ability to disrupt the basal lamina, using this preparation to assay the results. The effects of collagenase, chymotrypsin, elastase, ficin, papain, and trypsin on the integrity of the epithelium and the basal lamina are summarized below.

\section{Effects of enzymatic digestion on limb tissue}

The enzyme concentration range in which the limb epithelium remained intact and apparently healthy for $2-3 \mathrm{hr}$ was deter- 

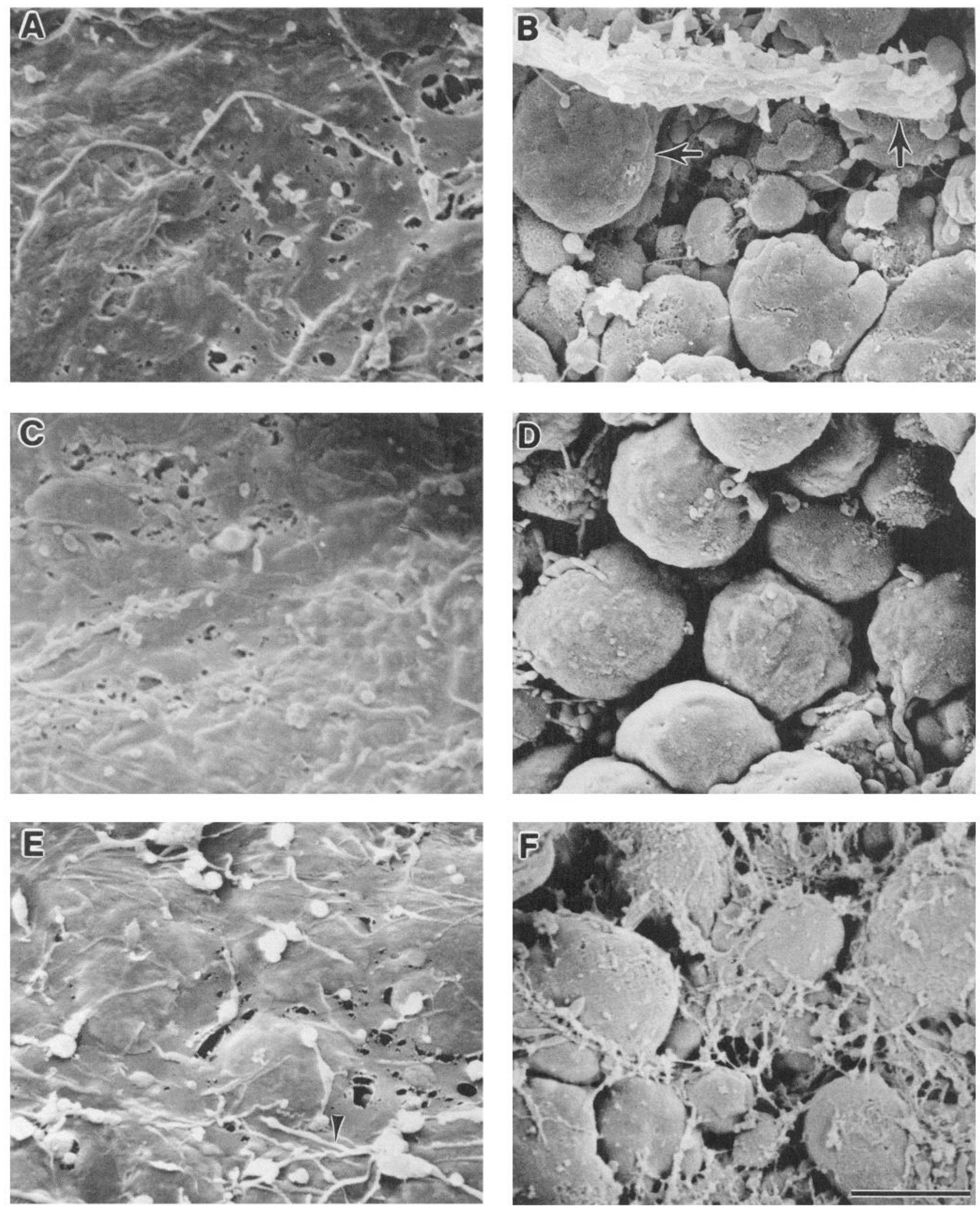

Figure 2. Scanning electron micrographs of the lumenal surface of limb epithelium after enzymatic treatment in limbs at the $32.5 \%$ stage of development. Higher-magnification views of the midfemur region in preparations similar to that seen in Figure $1 B$. $A$, Control limb showing foliose basal lamina completely obscuring the epithelial endfeet. B, Limb from the same clutch of eggs as control, incubated with $0.1 \%$ elastase for $2.5 \mathrm{hr}$. The basal lamina is completely removed. Horizontal arrow indicates epithelial basal endfoot; vertical arrow, a distal process of a Til neuron. $C$, Limb from the same clutch of eggs as control, incubated with $0.01 \%$ trypsin for $2.5 \mathrm{hr}$. The basal lamina remains intact, and similar in appearance to control. $D$, Limb treated with $0.02 \%$ ficin for $1.5 \mathrm{hr}$. The basal lamina is completely removed. $E$, Limb after treatment with $0.005 \%$ chymotrypsin for $2.5 \mathrm{hr}$. The basal lamina remains intact and similar in appearance to control. Mesodermal processes remaining after removal of the mesodermal cells are seen at the arrow. F, Limb incubated with $0.025 \%$ papain for $2.5 \mathrm{hr}$. The basal lamina is largely removed, although some fibrous material remains. Scale bar, $5 \mu \mathrm{m}$. 

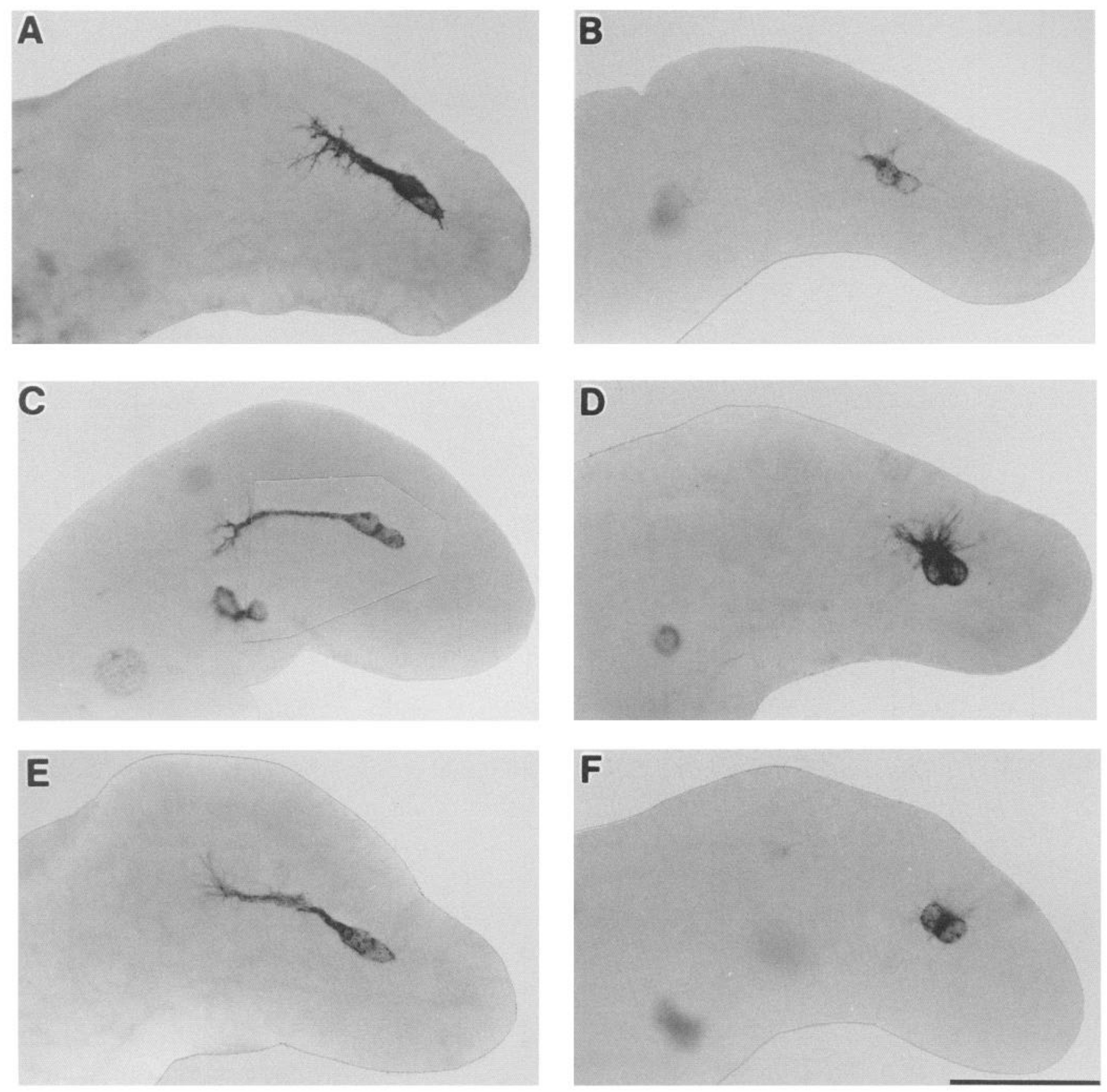

Figure 3. Til neurons in $32 \%$ stage limbs after enzymatic treatment. The Til neurons are labeled with anti-HRP antibodies, and viewed in wholemounted limbs. $A$, Control limb in which the axons have extended to approximately the midpoint of the femur. $B$, Limb that was incubated with $0.04 \%$ elastase for $2.5 \mathrm{hr}$. The Til growth cones have collapsed to the somata. Note the condensed mass of cellular material at the proximal pole of the Til cell pair. This prothoracic limb has elongated mildly so that it is approximately equal in length to a metathoracic limb (cf. $A$ ). $C$, Til neurons after incubation with $0.01 \%$ trypsin for $2.5 \mathrm{hr}$. The axons remain extended after enzymatic treatment. $D$, Til neurons after incubation with $0.02 \%$ ficin for $2.5 \mathrm{hr}$. The growth cones have collapsed to the somata. $E$. Til neurons after treatment with $0.005 \%$ chymotrypsin for $2.5 \mathrm{hr}$. The axons remain extended with numerous filopodia. $F$, Til neurons after exposure to $0.025 \%$ papain for $2.5 \mathrm{hr}$. The growth cones and axons have withdrawn. Dorsal is $u p$; distal is to the right. $A$ and $E$, metathoracic limb; $B, C$, and $F$, prothoracic limb; $D$, mesothoracic limb. Scale bar, $100 \mu \mathrm{m}$.

mined for each enzyme (see Materials and Methods and Table 1). The treatments do not affect the viability of the limb epithelium or the Til neurons since limbs cultured after enzymatic digestion continue to undergo epithelial morphogenesis and the Til neurons extend axons at rates approximately equal to control values (Table 2; M. L. Condic and D. Bentley, unpublished observations).

\section{Effects of enzyme treatment on the basal lamina}

The effects of different protease treatments on the basal lamina were investigated with scanning electron microscopy. Elastase, ficin, and papain had pronounced effects on the structural integrity of the basal lamina, resulting in either complete or partial degradation (Fig. 2, B, D, F; Table 1). Trypsin and chymotrypsin 
in contrast, had little to no effect on the structural integrity of the lamina (Fig. 2, $C, E$; Table 1). Incubation with 2 collagenases (cither Sigma C-0773 or one purified preparation) at the $32 \%$ stage had no consistent effect on either the integrity of the epithelium or the basal lamina at concentrations up to $2.0 \%$ and incubation times up to $6 \mathrm{hr}$. The effects of elastase, ficin, and trypsin on the basal lamina of older embryos were also observed (Table 1). For all ages inspected, elastase and ficin completely removed the basal lamina from all positions in the limb.

The time required for complete removal of the basal lamina in intact legs was increased considerably by the time required for diffusion of the bath solutions into the lumen of the leg. In limbs that had the mesoderm removed and/or were split open longitudinally in preparation for SEM prior to enzyme incubation, the time required both to obscrve effects on the Til neurons and to completely remove the basal lamina was decreased to as little as $1-1.5 \mathrm{hr}$.

\section{Retraction of growth cones in intrasegmental regions}

At the $32 \%$ stage of development. the Til neurons have extended axons several cell diameters in length in all 3 thoracic limbs and their growth cones have reached the midpoint of the femur (Keshishian and Bentley, 1983; Fig. 3A). At this stage, the Til growth cones are primarily in contact with intrasegmental epithelium and are near the location at which the first guidepost cell, the Fel neuron, will arise. There is, however, considerable variation in the time at which the $\mathrm{Fel}$ neuron arises relative to the time of Til outgrowth (Caudy and Bentley, 1986a).

When limbs at this stage of development were treated with elastase, ficin, or papain at concentrations shown to remove the basal lamina, the Til growth cones retracted partially or completely to the cell bodies (Table 1; Fig. 3, $B, D, F$ ). This growth cone retraction appeared to involve a progressive loss of substrate attachment since at lower concentrations or shorter incubation periods, axons were extended but devoid of filopodia and branches. Cells with retracted axons could be clearly distinguished from cells at a younger stage of development with axons of a similar length: Retracted axons showed a characteristic condensed mass of cellular material at the proximal pole of the Til cell pair or in the region of the growth conc (see Fig. $3 B$ ). Digestion with either trypsin or chymotrypsin did not result in growth cone retraction (Table 1; Fig. $3, C, E$ ). While the concentrations of enzyme chosen for these experiments intentionally resulted in a mild digestion, the effects of all enzymes on the neurons were also consistent at higher concentrations: Enzymes reported to have no effect on the neurons at the standard concentration had no effect even at concentrations sufficient to seriously compromise the integrity of the epithelium; cnzymes that did affect the disposition of the neurons showed a concentration- and time-dependent increase in both the incidence and magnitude of these effects.

\section{Quantification of growth cone retraction}

To quantify this effect, a clutch of eggs was sclected in which the Til axons had grown to the midpoint of the femur, but the Fel guidepost neuron was not yet evident either by staining with the anti-HRP antibody or by a response of the growth cones to an unlabled cell in the position of Fel (Caudy and Bentley, 1986b). Two chemically related enzymes with different effects on the basal lamina (elastase and trypsin, both serine proteases) were chosen to quantify the response of the Til growth cones to enzymatic treatment. Embryos were exposed to either

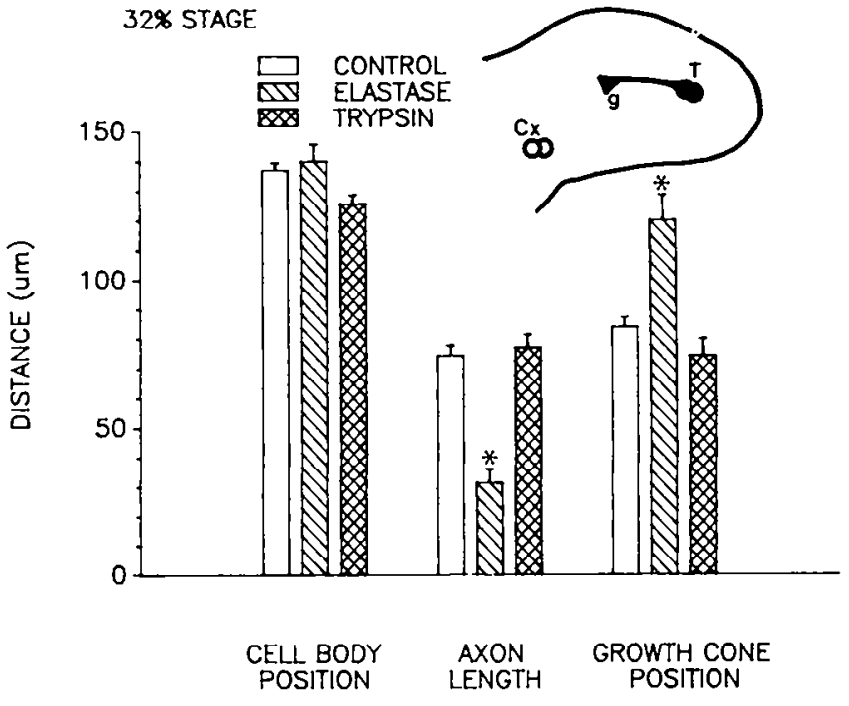

Figure 4. Quantification of neuron disposition after incubation with elastase and trypsin. Measurements were made on anti-HRP antibody labeled, whole-mounted embryos taken from a single clutch of eggs. Embryos at the $32 \%$ stage of development were exposed for $2.5 \mathrm{hr}$ to either saline (control, number of axon pairs $=40), 0.04 \%$ elastase $(n=$ 19 ) or $0.01 \%$ trypsin $(n=23)$. (ell body position is the distance between Til somata $(T)$ and $C x$ cells (see inset). Axon length is the distance from the growth cones $(g)$ to $\mathrm{T}$. Growth cone position is the distance between the growth cones and the $C x$ cells. The group means (har) and SEM (T) are presented. The asterisk indicates a significant difference from controls at $p<0.0001$, ANOVA.

elastase, trypsin, or saline, then fixed and labeled with anti-HRP antibodies. The length of the axon, the distance from the cell bodics of the Til neurons to the $\mathrm{Cxl}$ neurons, and the distance from the Til growth cones to the Cxl cells were measured for all 3 conditions. These results are summarized in Figure 4. Treatment with elastase, but not with trypsin, resulted in a significant decrease in axon length and a corresponding increase in the distance between the growth cones and the Cxl ncurons. These observations suggest that removal of the basal lamina with elastase results in retraction of the Til growth cones when the growth cones are extended over intrasegmental epithelium but are not yet in contact with the Fel neuron.

\section{Til growth cone response to sequential elastase treatments}

Growth cone retraction after protease digestion was observed with 3 different proteolytic enzymes known to remove the basal lamina, and not seen after treatment with 2 enzymes that leave the basal lamina intact. However, based on this correlation alone we could not discount the possibility that the response of the Til cells was due to general proteolysis of cell surface components rather than the removal of the basal lamina specifically. We therefore examined the effects of a second clastase digestion on Til axons that had extended in limbs after the basal lamina had becn removed by an initial elastase treatment. Under these conditions, the amount of basal lamina available as an enzymatic substrate during the second protease treatment should be greatly reduced relative to intact limbs. Consequently, proteolysis of neural and epithelial cell surface components should be much more pronounced during the second elastase digestion. Embryos at the $30 \%$ stage (prior to axon outgrowth) werc incubated with either saline or $0.04 \%$ elastase for $3 \mathrm{hr}$, rinsed extensively, and allowed to extend axons in culture for 16-17 

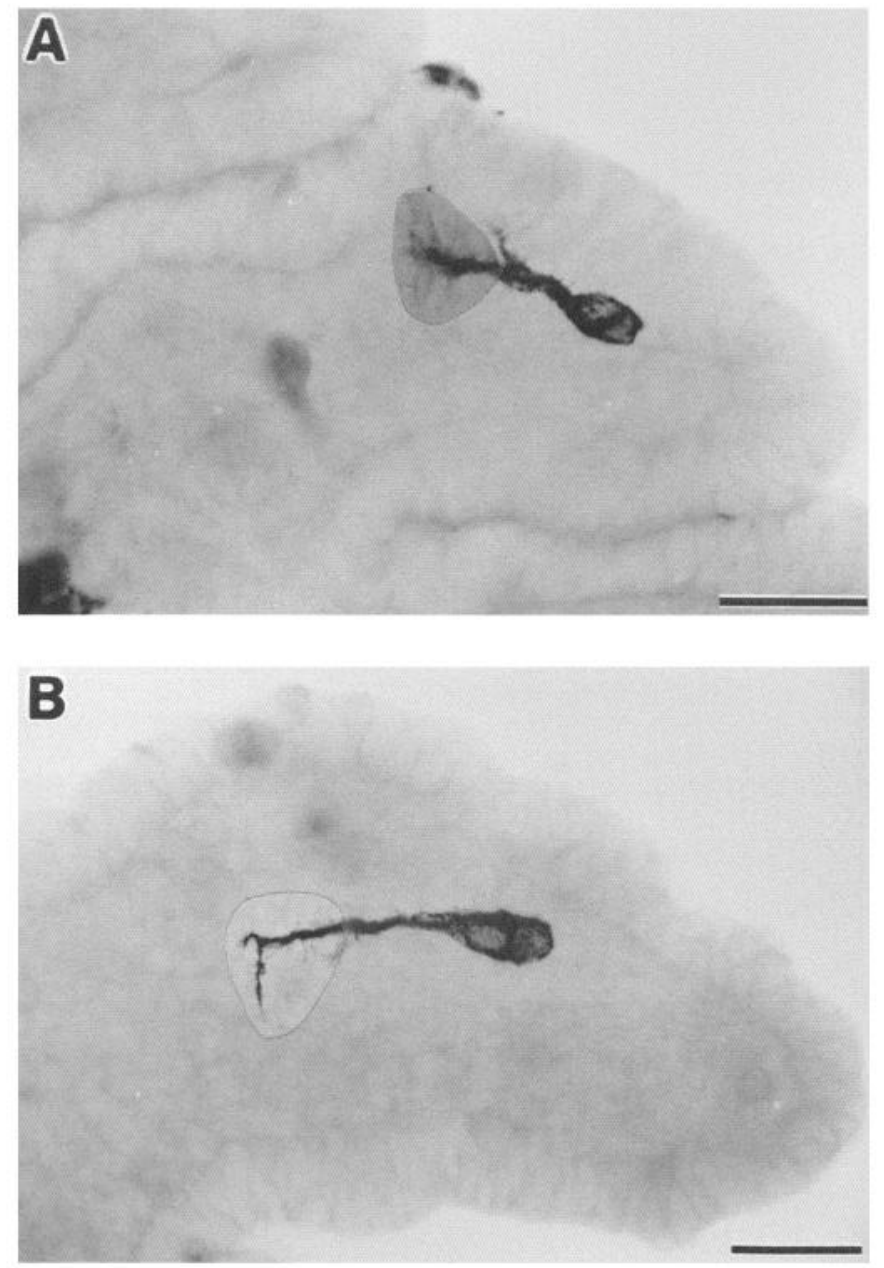

Figure 5. Til neurons in $32 \%$ stage limbs after elastase treatment in the presence of cytochalasin $\mathrm{D}$. The embryos were incubated with $0.04 \%$ elastase and $0.1 \mu \mathrm{g} / \mathrm{ml}$ cytochalasin D for $3 \mathrm{hr}$, then fixed. The Til neurons are labeled with anti-HRP antibodies, and viewed in wholemounted limbs. $A$, A prothoracic limb in which the axons and branches remain extended after treatment. $B$, A metathoracic limb also with extended axons after elastase and cytochalasin treatment. Almost all filopodia are withdrawn. (NB. The ventral branch is not on a limb segment boundary.) Dorsal is $u p$; distal is to the right. Scale bar, $50 \mu \mathrm{m}$.

hr. The numbers of limbs with axons greater than one cell diameter in length were approximately equal in control and enzyme-treated animals (Table 2; Condic and Bentley, unpublished observations). At the end of the culture period, both control and elastase-treated embryos were given a $3 \mathrm{hr}$ incubation with $0.04-0.01 \%$ elastase, and the response of the Til growth cone was observed. These results are summarized in Table 2. The axons of cultured control embryos responded to removal of the basal lamina with elastase by retracting, as anticipated from earlier results. In contrast, axons that had extended after the basal lamina had been removed by an initial elastase treatment did not retract after a second elastase treatment.

\section{Growth cone response to elastase treatment in the presence of cytochalasin}

Axon retraction could be caused by tension in the actin microfilament cortex (Joshi et al., 1985; Letourneau et al., 1987). The identity of the cytoskeletal component(s) mediating Til growth cone retraction after removal of the basal lamina was investigated using a microfilament-depolymerizing agent, cytochalasin D. Elastase digestions were conducted in the presence of cytochalasin D, and the response of the Til growth cones was observed in anti-HRP labeled whole-mounts. In the presence of cytochalasin D, removal of the basal lamina by elastase did not result in the retraction of the Til growth cones (Fig. 5A). The Til axons often appeared devoid of filopodia (Fig. $5 B$ ), but remain extended after elastase treatment. These results are summarized in Table 3 . Incubation with cytochalasin D alone or in combination with elastase increased the frequency of withdrawn axons relative to control values. However, the frequency of axon retraction observed with elastase treatment alone is more than twice that seen with elastase in the presence of cytochalasin D. These results suggest that the Til axons are under tension in vivo, and that the growth cone retraction observed after removal of the basal lamina with elastase is mediated by actin microfilaments.

\section{Discussion}

In the work reported here we have shown that the basal lamina can be removed from embryonic grasshopper limb buds by mild enzymatic treatment. We have used this procedure to analyze the nature of growth cone-basal lamina interdependence in vivo. We have shown that the Til axons are under tension and that adhesive interactions of the Til growth cones underlie axon outgrowth in vivo.

\section{Removal of the basal lamina}

We have demonstrated that incubating grasshopper limb buds with elastase, ficin, or papain results in substantial or complete removal of the basal lamina, whereas trypsin and chymotrypsin have mild to negligible effects on the basal lamina (see Fig. 2, Table 1). In contrast to most vertebrate tissue, the basal lamina of insects is generally quite resistant to removal by enzymatic treatment. Digestion with hyaluronidase, collagenase, and chymotrypsin alone or in combination was insufficient to remove the basal lamina from Malpighian tubules of Rhodnius (Satmary and Bradley, 1982). The use of a mild elastase treatment to digest extracellular material has been reported previously in insects (Baccetti and Bigliardi, 1969; Locke and Huie, 1972). Elastase removes the basal lamina from Rhodnius, Drosophila (Levinson and Bradley, 1984), and Tenebrio (Koefoed, 1987) epithelia. Elastase has also been used successfully to dissociate embryonic grasshopper tissue for cell culture (Lefcort et al., 1986). The observation that the basal lamina of grasshopper limbs can also be removed by treatment with 2 plant thiol proteases (papain and ficin) while enzymes more closely related to elastase (trypsin and chymotrypsin) are relatively ineffective is somewhat surprising. All 5 enzymes used in this study are general proteases with broad specificities and different preferred cleavage sites. However, elastase, ficin, and papain are known to digest at least one extracellular protein against which trypsin and chymotrypsin are relatively inactive (Thomas and Partridge, 1960). Elastase has been shown to degrade the lamininnidogen complex of murine tumor basement membrane (Paulsson et al., 1987). Basal laminae of insects contain both laminin (Fessler et al., 1987; Montell and Goodman, 1988) and an entactin/nidogen-like compound (Blumberg et al., 1987).

In our experiments, removal of the basal lamina by treatment with elastase left the tissue intact and viable, as evidenced by the growth and differentiation of both epithelium and neurons 
in whole embryo culture after enzymatic treatment (Table 2; Condic and Bentley, 1986, 1988; Condic and Bentley, unpublished observations). Results from dissociated culture of grasshopper cells also suggest that elastase treatment does not affect the viability of neurons; if embryonic tissue is dissociated with relatively high concentrations of elastase $(0.4 \%)$, numerous neurons capable of extending long axons are observed in culture (Lcfcort ct al., 1986). These results are consistent with observations of elastase-treated insect tissue in other systems. After removal of the basal lamina with elastase, epithelial tissue of Malpighian tubules in Rhodnius and imaginal disks in Drosophila remained intact and viable based on ultrastructural criteria and trypan blue exclusion (Levinson and Bradley, 1984). Removal of the basal lamina by elastase from Tenebrio larvae did not affect the epithelial ultrastructure or the ability of the intact epithelia to maintain pretreatment intracellular concentrations of sodium and potassium ions (Koefoed, 1987).

\section{Axons under tension in vivo}

If the Til growth cones had extended in the presence of an intact basal lamina, removing the basal lamina caused the growth cones to retract to the cell somata (see Fig. 3 ). This retraction could be inhibited by cytochalasin $\mathrm{D}$, suggesting that microfilament-based tension underlies the response. The changes in Ti1 growth cone position occurred relatively rapidly (within $1.5 \mathrm{hr}$, allowing an estimated $1 \mathrm{hr}$ for enzyme diffusion into the limb) and involved a substantial decrease in the average length of the Til axons. These data strongly suggest that the Til axons are under tension in vivo.

There is considerable evidence in support of the idea that axons are under tension when grown in vitro. The branching patterns of neurites produced by chick dorsal root ganglion cells in culture are consistent with tension being exerted on the neuronal fibers by their growth cones (Bray, 1979). In PC 12 cells, neurites retract to the cell somata within 2 min after being detached from the substratum by laser transection. In addition, detachment of the growth cone results in the rapid translocation of the cell body to a new equilibrium position between the remaining neurites. These effects are inhibited by cytochalasin, strongly suggesting that neurite tension is supported by cytoskeletal actin filaments (Joshi et al., 1985). A similar inhibition of neurite tension is seen in chick dorsal root ganglion cells after treatment with cytochalasin B or D (Letourneau et al., 1987). In primary cultures of chick sensory neurons, severed neurites either remain extended or rapidly retract after amputation, depending on the adhesivity of the substratum (Baas et al., 1987). The retraction of the Til growth cones observed after enzymatic treatment suggests that the Til axons are under tension in vivo and that removing the basal lamina decreases the adhesion of the growth cones to the substratum. The implications of these results for neural-basal lamina adhesion are discussed below.

\section{Neural-basal lamina adhesion}

Although immature neurons and limb segment boundaries are important growth cone guidance cues in this system, in the initial stages of axonal outgrowth (30-32\% of development), the Til growth cones are neither in contact with other neurons nor with a well-developed segment boundary. Limbs at this stage of development were selected to assess whether Til growth cones that had extended in the presence of an intact basal lamina are dependent on the basal lamina to remain extended within this intrasegmental epithelial region.
Our results demonstrate that some proteolytic digestions result in growth cone retraction at this stage, whereas others do not. Neither trypsin nor chymotrypsin significantly disrupts the basal lamina, and growth cone retraction was not consistently observed with either of these enzymes. This stands in contrast to the relative ease with which trypsin induces retraction of vertebrate neurites in vitro (Letourneau et al., 1987). Changes were observed in Til growth cone position after treatment with 3 different enzymes (elastase, ficin, and papain), each of which also has a pronounced effect on the integrity of the basal lamina. The correlation of presence or absence of the basal lamina with changes in neuronal extension under a variety of enzymatic conditions suggests that a structurally intact basal lamina may be sufficient to constrain the position of the Til growth cones and somata in vivo. It is unlikely that an intact basal lamina is a necessary condition for neuronal substrate adhesion in this system since the Til neurons are capable of extending axons over an epithelium stripped of basal lamina by elastase (Table 2; Condic and Bentley, unpublished observations). Some of the decrease in Til substrate adhesion observed after treatment with elastase, ficin, and papain might be due to degradation of epithelial and/or neuronal cell surface proteins in addition to the effect of these enzymes on the basal lamina. However, when axons extend in limbs from which the basal lamina has been removed by elastase treatment, they do not retract in response to a second, equivalent protease treatment. Proteolytic degradation of cell surface proteins should be even more pronounced during the second protease digestion. If growth cones advance by adhering to basal processes of epithelial cells, disruption of epithelial-basal lamina adhesion could indirectly interfere with growth cone-epithelial cell adhesion; retraction of epithelial endfeet could mechanically disrupt growth cone-epithelial adhesion. However, Ti1 growth cones are able to extend over epithelial cells after removal of the basal lamina, suggesting that epithelial-basal lamina interactions are not necessary for neurite extension. Since growth cone-epithelial interactions after removal of the basal lamina must be sufficiently adhesive to resist axonal tension, it is unlikely they would be easily dislodged. Moreover, adhesive interactions between Til growth cones and either neurons or epithelial segment boundary cells are able to resist considerable mechanical force (Condic and Bentley, 1989). These results suggest that under normal conditions of neurite outgrowth (when the axons have extended in the presence of an intact basal lamina), neurites are dependent on direct, adhesive interactions with the basal lamina to remain extended within the intrasegmental region.

Neuronal growth cones interact with a variety of basal lamina associated proteins, and many of these interactions appear to be adhesive in nature (Jessell, 1988). There is evidence that growth cone-basal lamina adhesive interactions serve to guide extending neurites in vitro (Hammarback et al., 1988). Growth cone withdrawa! in response to removing the basal lamina suggests that the Til neurons extend in vivo by preferentially using basal lamina components as a substrate. Growth cone-substrate interactions in this system are at least in part adhesive, as evidenced by the ability of these interactions to resist axon tension.

\section{References}

Ashhurst, D. E. (1965) The connective tissue sheath of the locust nervous system: Its development in the embryo. Q. J. Microsc. Soc. 106: 61-73.

Ashhurst, D. E. (1982) The structure and development of insect con- 
nective tissues. In Insect Lltrastructure, R. C. King and H. Akai, eds., pp. 313-350, Plenum, New York.

Baas, P. W., L. A. White, and S. R. Heidemann (1987) Microtubule polarity reversal accompanies regrowth of amputated neurites. Proc. Natl. Acad. Sci. USA 84: 5272-5276.

Baccetti, B., and E. Bigliardi (1969) Studies on the fine structure of the dorsal vessel of arthropods. I. The 'heart' of an orthopteran. $Z$. Zellforsch. 99: 13-24.

Ball, E. E., and C. S. Goodman (1985) Muscle development in the grasshopper embryo. Il. Syncytial origin of the extensor tibiac muscle pioneers. Dev. Biol. 111: 399-416.

Ball, E. E., H. G. deCouet, P. L. Horn, and J. M. A. Quinn (1987) Haemocytes secretc basement membrane components in embryonic locusts. Development 99: 255-259.

Bate. C. M. (1976) Pioneer neurons in an insect embryo. Nature 260: $54-56$

Bentley, D., H. Keshishian, M. Shankland. and A. Toroian-Raymond (1979) Quantitative staging of embryonic development of the grasshoppcr. Schistocerca nitens. J. Embryol. Exp. Morphol. 54: 47-74.

Blumberg. B., A. J. MacKrell, P. F. Olson, M. Kurkinen, J. M. Monson, J. E. Natzle, and J. H. Fessler (1987) Basement membrane procollagen IV and its specialized carboxyl domain are conserved in Drosophila, mouse and human. J. Biol. Chem. 262: 5947-5950.

Bogacrt, T., N. Brown, and M. Wilcox (1987) The Drosophila ps2 antigen is an invertebrate integrin that, like the fibronectin receptor, becomes localized to muscle attachments. Cell 51: 929-940.

Bray, D. (1979) Mechanical tension produced by nerve cells in tissue culture. J. Cell Sci. 37: 391-410.

Caudy, M., and D. Bentley (1986a) Pioneer growth cone morphologies reveal proximal increases in substrate affinity within leg segments of grasshopper embryos. J. Neurosci. 6: 364-379.

Caudy, M., and D. Bentley (1986b) Pioneer growth cone stecring along a series of neuronal and non-neuronal cues of different affinities. $J$. Neurosci. 6: 1781-1795.

Condic, M. L., and D. Bentley (1986) Effects of proteolytic, glycolytic and basal lamina-directed enzymes on pathfinding by grasshopper pionecr neurons. Soc. Neurosci. Abstr. 12: 194.

Condic, M. L., and D. Bentley (1988) Effects of enzymatic removal of the basal lamina on pioneer neurons in grasshopper embryos. Soc. Neurosci. Abstr. 14:451.

Condic, M. L., and D. Bentley (1989) Pioncer growth cone adhesion in vivo to boundary cells and neurons after enzymatic removal of basal lamina in grasshopper embryos. J. Neurosci. 9: 2687-2696.

Fessler, L. I. A. G. Campbell, K. G. Duncan, and J. H. Fessler (1987) Drosophila laminin: Characterization and localization. J. Cell. Biol 105: 2383-2391.

Grateios, D., C. Naidet, M. Astier, J. P. Thiery, and M. Semeriva (1988) Drosophila fibronectin: A protein that shares properties similar to those of its mammalian homologue. ЕMBO J. 7: 215-223.

Hammarback, J. A., J. B. McCarthy, S. L. Palm, L. T. Furcht, and P. C. Letourneau (1988) Growth cone guidance by substrate-bound laminin pathways is correlated with neuron-to-pathway adhesivity. Dev. Biol. 126: 29-39.

Hay. E. D. (1981) Collagen and embryonic development. In C'ell Biology of Extracellular Matrix, E. D. Hay, ed., pp. 379-409, Plenum, New York.

Hynes, R. O. (1987) Integrins: A family of cell surface receptors. Cell 48: $549-554$

Jan, L. Y., and Y. N. Jan (1982) Antibodies to horseradish peroxidase as specific neuronal markers in Drosophila and grasshopper embryos. Proc. Natl. Acad. Sci. USA 79: 2700-2704.

Jessell. T. M. (1988) Adhesion molecules and the hierarchy of neural development. Neuron $1:$ 3-13.
Joshi, H. C., D. Chu, R. E. Buxbaum, and S. R. Heidemann (1985) Tension and compression in the cytoskeleton of PC 12 ncurites. J. Cell. Biol. 101: 697-705.

Keshishian, H. (1980) The origin and morphogenesis of pioneer neurons in the grasshopper metathoracic leg. Dev. Biol. 80: 388-397.

Keshishian, H., and D. Bentley (1983) Embryogenesis of peripheral nerve pathways in grasshopper legs. Dev. Biol. 96:98-124.

Koefoed. B. M. (1987) The ability of an epithelium to survive removal of the basal lamina by enzymes: Fine structure and content of sodium and potassium of the midgut epithelium of the larva of Tenebrio molitor after withdrawal of the basal lamina by elastase - a short note. Tissue Cell 19:65-70.

Lefcort, F., and D. Bentley (1987) Pathfinding by pioneer neurons in isolated, opened and mesoderm-free limb buds of embryonic grasshoppers. Dev. Biol. 119: 466-480.

Lefcort, F., and D. Bentley (1989) Organization of cytoskeletal elements and organelles preceding growth cone initiation in an identified neuron in situ. J. Cell. Biol. 108: 1737-1749.

Lefcort. F., M. L. Condic, and D. Bentley (1986) Recognition between grasshopper afferent neurons in vitro. Soc. Neurosci. Abstr. 12: 196.

Leptin, M., R. Aebersold, and M. Wilcox (1987) Drosophila positionspecific antigens resemble the vertebrate fibronectin-receptor family. EMBO J. 6: 1037-1043

Letourneau, P. C. T. A. Shattuck, and A. H. Ressler (1987) "Pull" and "push" in neurite elongation: Observations on the effects of different concentrations of cytochalasin b and taxol. Cell Motil. Cytoskel. 8: 193-209.

Levinson, G., and T. J. Bradley (1984) Removal of insect basal laminac using elastase. Tissuc Cell 16:367-375.

Locke, M., and P. Huie (1972) The fiber components of insect connective tissue. Tissue Cell 4: 601-612.

MacKrell, A. J., B. Blumberg, S. R. I layncs, and J. H. Fessler (1988) The lethal myospheroid gene of Drosophila encodes a membrane protein homologous to vertebrate integrin B subunits. Proc. Natl. Acad. Sci. USA 85: 2633-2637.

Mirre, C., J.-P. Cecchini, Y. Le Parco, and B. Knibiehler (1988) De novo expression of a type IV collagen gene in Drosophila embryos is restricted to mesodermal derivatives and occurs at germ band shortening. Development 102: 369-376.

Montell, D. J., and C. S. Goodman (1988) Drosophila substrate adhesion molecule: Sequence of laminin $\mathrm{Bl}$ chain reveals domains of homology with mouse. Cell 53:463-473.

Obrink, B. (1986) Epithelial cell adhesion molecules. Exp. Cell Res 163: $1-21$

Paulsson, M.. M. Aumailley, R. Deutzmann, R. Timpl. K. Beck, and J. Engel (1987) Laminin-nidogen complcx: Extraction with chelating agents and structural characterization. Eur. J. Biochem. 166: 11-19.

Sanes, J. R., and A. Y. Chiu (1983) The basal lamina of the neuromuscular junction. Cold Spring Harbor Symp. Quant. Biol. 48: 667678.

Satmary, W. M., and T. J. Bradley (1982) Dissociation of insect malpighian tubules into single, viable cells. Am. Zool. 22: 914.

Snow, P. M., N. H. Patel, A. L. Harrelson. and C. S. Goodman (1987) Neural-specific carbohydrate moiety shared by many surface glycoproteins in Drosophila and grasshopper embryos. J. Ncurosci. 7: 41374144.

Thomas, J.. and S. M. Partridge (1960) The chemistry of connective tissues: The elastase activity of protcolytic enzymes. Biochem. J. 74: 600-607.

Wigglesworth. V. B. (1953) The origin of sensory neurones in an insect Rhodnius prolixus (Hemiptera). Q. J. Microsc. Sci. 94: 93-112. 